\title{
Stress Management in Primary Caregivers: A Health Challenge
}

\author{
Sira Aguiló ${ }^{1}$, Beatriz Lopez-Barbeito ${ }^{1}$, Esther García ${ }^{3}$, Jordi Aguilón \\ ${ }^{1}$ Hospital Clínic. Barcelona, Spain \\ ${ }^{2}$ Department of Microelectronics and Electronic Systems, Universidad Autónoma de Barcelona, \\ Barcelona, Spain \\ ${ }^{3}$ Biomedical Research Networking Center (CIBER-BBN), Spain \\ acmlis.conference@gmail.com
}

\begin{abstract}
Technology based advances in healthcare are leading to social changes that, in turn, will require innovative responses from health systems. Mainly due to these advances, life expectancy grows so, the aging of world population increases and with it the percentage of chronic patients. Because ofthese two factors: aging people and chronic patients, new needs are posed to the current health system. Together with these patients, a population of non-professional caregivers emerges playing a key role in the care process. However, the impact of the care burden on their health is usually underminedalthough an increasing number of studies report caregiver associated diseases. One of the challenges that arise from health systems: To acknowledgeand to take care this population of caregivers, to avoid the so-called burned caregiver syndrome as well as to reduce the risks of comorbidities associated with the caregiver's stress by setting up appropriate interventions.

Having in mind all these considerations, from the Clinic Hospital in Barcelona we are starting to work ona biofeedback program involvingdifferent populations of caregivers: 30 caregivers of oncological patients and 30 caregivers of geriatric patients and the corresponding control group. The project that is presented in this paper is aiming to determine if these therapies can become effective interventions to reduce the stress that represents the caregiver syndrome. The follow-up of the project willfocus on the possibility to indirectly demonstrate if the decrease of stress actually results in a decrease of the derived diseases and of the total cost that it entails.
\end{abstract}

Keywords: Primary caregivers, Caregiver's syndrome, Stress Management, Biofeedback

\section{Introduction}

The increase in life expectancy and in population aging together with medical and healthcare advances are leadingtowards a big change in the world health scenario that will dramatically change the starting point of the public health systems and of course his primary needs to face these new circumstances. The longevity of patients with chronic diseases such as Chronic Obstructive Pulmonary Disease COPD, heart failure or dementia as well as the chronicity of hemato-oncological processes that until a few years ago lacked of treatment have revealed the need to implementsupport units, management and monitoring of *this group of chronic patients. 
In parallel, a population of caregivers and primary caregivers of these chronic patients emerges as an indispensable help for the management and accompaniment of this kind of patients while in fact are solving problems and responsibilities of the public health system. Primary caregiver is defined as the individual acting as caregiver of the patient for a minimum of 6 weeks, without receiving financial compensation, with direct emotional involvement and living at home with the patient [1][2]. Primary caregiver is thus subjected to emotional and physical stress derived from the objective burden of the tasks required for patient's care and normally the financing of a significant part of the care expenses and of course the emotional load that all of this represents [3] [4].

In Spain, the economic impact derived from care was1.7-4.9\% of Spanish Gross Domestic Product,(GDP) in 2008 [4] while the need for geriatric patient'scaregivers will be about 2.5 million people by 2060 [5]; and this emerging population of caregivers will be subjected to an already recognized physical and psychological stress with its long-term health implications.

From an emotional point of view, usuallycaregivers are not sure about their abilities for the management of the patient,about the best strategy to improve the patient wellbeing or about the better way to achieve theirobjectives [6].Over time, this leads to frustration, anxiety, depression and associated comorbidities such as diabetes, the increase of their cardiovascular risk factor, hypertension or dyslipidemia[7][8] among others which will affect their own health and therefore health and wellbeing of the patient they are in charge of.

From a physiologicalpoint of view, this population of primary caregivers is affected by a deterioration oftheir perceived physical and mental health [9] andoftheir immune system response,they are prone to increase drug consumption[10] and more than half of them will show headache episodes and musculoskeletal problems [11][12]. Moreover, caregivers older than 65 years old who care for their dependent partner have a statistically significant higher mortality risk compared to non-caregivers [13].

The approach to this population of caregivers was initiated years ago, focusing on the collective caring for people in the geriatric age and with a physical and cognitive deterioration.Throughout the last decades some tools have been designed to evaluate the degree of caregiver overload. Among them the Zarit (EZ) Caregiver Overload Scale [14] and The Caregiver Strain Index, which consists of 13 items with yes / no binary responses and where a high level of stress is considered if the caregiver score is greater than 7.

Other initiatives such as the Infosa "Caring for the caregiver" [15] showed that group sessions of empowerment reduced the perception of stress in a population of caregivers of geriatric patients with chronic illnesses.

We are facing a new health challenge: addressing the needs of a growing population of caregivers and proposing preventive measures so that the caregiver's own stress does not lead to long-term illness. The challenge is not straightforward, since so farthere is no consensus about astress measurement method.

Although the WHO has described stress as the new epidemic of s. XXI, several measuring approachesare advancing slowly and still in a very subjective way being the psychometric tests the current gold standard for stress diagnosis and evaluation.

Another approach consideringthe physiological responses of the hypothalamic-pituitary-adrenal system(HPA), and the autonomic nervous system(AN), as well as the different perceptions against the 
Sira Aguiló, Beatriz Lopez-Barbeito, Esther García, Jordi Aguiló. Stress Management in Primary Caregivers: A

Health Challenge. Transactions on Machine Learning and Artificial Intelligence, Vol 5 No 4 August (2017);

p: 558-564

same or similar stimulus, has high inter-individual variations that make it difficult to establish a quantitative assessment methodorstrategies proposedfor their treatment.

On the other hand, in recent years newprocedures with biofeedback techniques have impacted on the treatment of diseases such as asthma [16], AHT, anxiety or duodenal ulcer [17].Biofeedback techniques consist in giving feedback to the individual with information from hisown physiological variables, such as heart rate, respiratory rate, or muscle contraction force, to know and to learn how these variables can be controledand hence gaininga better control of his own biological responses. In this way, learning and getting control of their own responses we can modulate the AN balance by achieving a greater activity of the parasympathetic system and a reduction of the sympathetic response with the consequent reduction of the physiological cascade generated as response to stress.

In this pilot study, we try to use biofeedback techniques to improve stress perception in a population of primary caregivers of patients with chronic disease and to evaluate their effectiveness according to quality of life indexes or measures, anxiety symptoms, depression and physiological variables such as HRV (heart rate variability) that have demonstrated their involvement in stress [18].

\section{Project Objective}

The main objective of the project is to determine if biofeed-back techniques are useful as tools to improve quality of life and reduce stress in a population of primary caregivers of chronic patients.

\section{Methodology and Experiment's Organization}

The experimental pilot study consists in the consecutive inclusion of60 primary caregivers of chronic patients that meet the primary caregiver definition: individual acting as caregiver of the patient for a minimum of 6 weeks, without receiving financial compensation, with direct emotional involvement and living at home with the patient

Two types of primary caregivers will be collected:

On the one hand, caregivers of geriatric patients with chronic disease usually chronic lung disease, heart failure or dementia, who require help for daily life activities due to functional or cognitive impairment.

On the other hand, selection includes caregivers of non-geriatric patients with active onco-hematologic processes, requiring a primary caregiver for agenda compliance, management of side effects of managed treatments and emotional accompaniment according the disease progress.

Included subjects will be cited to a first session, of initial evaluation, where the sociodemographic characteristics, indicated in table 1, and the psychometric tests, indicated in table 2, will be collectedThese tests can be self-administered.An approximate time of 20 minutes is calculated to finish the three tests proposed. However, tests are not time dependent and no fixed response time will be considered for psychometric evaluation.Heart Rate Variability, HRV, trapezoidal Electromyography, EMG, and Respiratory Rate, RR, will be recorded during 10 minutes through a standard electronic equipment NEXUS 10 Mark II, NX10B-EU.

After the initial evaluation session, five biofeedback sessions will follow according to the agreed agenda. These sessions will be devoted to learn and practice about breath control and muscle contraction. Sessions will be spread over five weeks and each lasting about 40 minutes. 
The sessions are individual with a therapist trained in biofeedback management.After each sessiona document with detailed information about a series of exercises that should practice at home for 10 minutes a day every day, will be provided. Home practice is to strengthen what was taught in the face-toface session.

After these five biofeedback sessions, in week 6, the subject will be newly evaluatedfollowing the same procedure than in initial session. That is, the psychometric tests will be repeated, the physical variables will be monitored and a quality of life questionnaire will be passed.

Information of the health status of the patient at week 6 will also be requested and collected regarding their initial state because the patient health statecan influence the stress and quality of life of their caregivers independently to the sessions given.

The results obtained before and after the biofeedback sessions will be compared in order tofindsignificant differences between the degree of stress according to the psychometric tests, the quality of life questionnaireas well as in the physiological signals recorded, HRV, RR, EMG.

\section{Material}

Continuous monitoring, conditioning and recording of signals will becarried out viaNEXUS 10 Mark II, NX1OB-EU de Min Media BV, Netherlands CE mark CE0344 that meetsall the requirements of the MDD (93/42/EEC). MDD Classification IIa and IEC 60601-1-6: 2004 Concerning Electromagnetic compatibility.The system has 12 operating channels in which different sensors can be connected. The main technical characteristics of the measurement device are shown in Table I.

Table 1. Electronic device characteristics

\begin{tabular}{|c|c|}
\hline \multicolumn{2}{|r|}{ Bipolar Inputs } \\
\hline Input signaldifference & $<0.1 \mathrm{Vpp}$ \\
\hline Input commonmoderange & $-2.0 \mathrm{~V} \ldots+2.0 \mathrm{~V}$ \\
\hline Gain factor & 19.5 \\
\hline Noise & $<1 \mu \mathrm{V}$ RMS $(1-64 \mathrm{~Hz}$ freq. range \\
\hline Input impedance & $>=1012$ Ohm (Instrumentation amp at DC) \\
\hline CMRR (typical) & $>=100 \mathrm{~dB}$ \\
\hline Accuracy & $+/-2 \%$ \\
\hline \multicolumn{2}{|r|}{ Auxiliary Inputs } \\
\hline Input signalrange & $-2.0 \mathrm{~V} \ldots+2.0 \mathrm{~V}$ \\
\hline Input commonmoderange & $-2.0 \mathrm{~V} \ldots+2.0 \mathrm{~V}$ \\
\hline Gain factor & 1 \\
\hline Noise & $<3 \mu \mathrm{V}$ RMS (1 - $64 \mathrm{~Hz}$ freq range) \\
\hline Input impedance & $>=1010$ Ohm (Instrumentation amp at DC) \\
\hline CMRR (typical) & $>=80 \mathrm{~dB}$ \\
\hline Accuracy & $+/-2 \%$ \\
\hline \multicolumn{2}{|r|}{ AD Conversion } \\
\hline Resolution & Bipolar $12.2 \mathrm{nV} / \mathrm{bit}$, auxiliary $0.238 \mu \mathrm{V} / \mathrm{bit}$ \\
\hline Samplefrequency (Bipolar) & $\begin{array}{l}8192 \mathrm{~Hz}, 4096 \mathrm{~Hz}, 2048 \mathrm{~Hz}, 1024 \mathrm{~Hz} \\
512 \mathrm{~Hz}, 256 \mathrm{~Hz}, 128 \mathrm{~Hz}\end{array}$ \\
\hline $\begin{array}{l}\text { Samplefrequency } \\
\text { (auxiliary) }\end{array}$ & $256 \mathrm{~Hz}, 128 \mathrm{~Hz}$ \\
\hline Channelbandwidth & DC up to 0.4 * sample frequency \\
\hline
\end{tabular}


Sira Aguiló, Beatriz Lopez-Barbeito, Esther García, Jordi Aguiló. Stress Management in Primary Caregivers: A

Health Challenge. Transactions on Machine Learning and Artificial Intelligence, Vol 5 No 4 August (2017);

p: $558-564$

The device is organized in 12 operating channels in which sensors could be connected. Resolutions and signal ranges are different depending on the channel number. There are 4 dedicated channels. Table II summarizes Chanel function, range and resolution.

\begin{tabular}{|l|l|l|l|}
\hline ChanelNr. & Function & Resolution/bit & Signalrange \\
\hline $1,2,3,4$ & Bipolar signals & $12.2 \mathrm{nV}$ & $\pm 100 \mathrm{mV}$ \\
\hline $5,6,7,8$ & Auxiliarysignals & $0.24 \mu \mathrm{V}$ & $\pm 2.0 \mathrm{~V}$ \\
\hline 9 & Oxygensaturation & $1 \%$ & $0-100 \%$ \\
\hline 10 & Plethysmographic & 1 (bit) & $0-255$ \\
\hline 11 & Pulseoximeterheartrate & 1 BPM & $0-255 \mathrm{BPM}$ \\
\hline 12 & Pulseoximeter status & 1 (bit) & $0-255$ \\
\hline
\end{tabular}

\section{Discussion}

Caregivers of chronic patients is an emerging population, with a role not always well defined, submitted to physical and emotional stress.Better management focusing on the stress reduction is important in mitigating long-term health consequences such as anxiety, depression, musculoskeletal problems or cardiovascular events, and to ensure good care of those who are in charge.

Although different subpopulations of caregivers have not yet been defined, it seems obvious that that there will be different groups of caregivers with different needs. As an example, it seems to be clear that caregivers of geriatric patients with cognitive impairment needing help for basic activities such as grooming or feeding are a population other than caregivers of autonomous patients diagnosed with an active hemato-oncological process.

The current health challenge in this field is in the context of an increasingly aging population, with chronic diseases and active onco-hematological processes that last over time.

This implies the need for a conceptual change, but also a practical one allowingto takecare of the needs of this group of patients, as well as of those of their caregivers, who are indispensable pieces of health processes.

It is well established that being a primary caregiver is stressful and that it has long-term consequences. However, interventions are difficult because of the interindividual differences in the perception and response to stress and the difficulty of not having a validated tool to quantify and / or measure the stress level.

Given the already stated sociodemographic evolution, to propose and to test strategies for improvement and control of the caregiver's stress and of its consequences should be a major health objective.

Biofeedback techniques seem to be a good tool for stress management and therefore, to use it as therapeutic strategy to improve quality of life in this population group. Once it is clear from publications [19] and from our own experience that the stress level could be quantitatively evaluated, what we are doing at the Clinic Hospital in Barcelona presented in this paper is to prove that biofeedback techniques could be an effective treatment to reduce the stress level. Additionally, to prove the efficacy of the applied techniques we will measure the quality of life,the QOL Index [20], using scales of perceived stress and using also the physiological variables that are constituting a stress biomarker. 
Although there are different studies approaching problems of the caregiver's population, most of them are merely descriptive and don't propose improvementstrategies or evaluate possible effects of the implementation of this kind of interventions in this group.

The characterization of the behavior of both groups of caregivers will allow to observe the different response to the proposed interventions. Which will allow the modeling of the system and offer customized interventions

\section{ACKNOWLEDGEMENTS}

This research line is supported by the Hospital Clinic de Barcelona, http://www.hospitalclinic.org/, the CIBER-BBN (http://www.ciber-bbn.es)and the Univ. Autónoma de Barcelona throughthe MINECO (FISPI12/00514 and TIN2014-53567-R)

\section{REFERENCES}

[1] [1] Mateo, I, Millán, A, García, M, Gutiérrez, P, Gonzalo, E. y López, L. A." Cuidadores familiares de personas con enfermedad neurodegenerativa: perfil, aportaciones e impacto familiar", Atención Primaria. $2000 ; 26 ; 25-34$.

[2] Rodríguez, P., Sancho, T., Alvaro, M. y Justel, M.” Las personas mayores en España. Perfiles. Reciprocidad familiar. Madrid: Instituto Nacional de Servicios Sociales

[3] Pinquart M, Sörensen S. "Associations of stressors and uplifts of caregiving with caregiver burden and depressive mood: a meta-analysis". J Gerontol B Psychol Sci Soc Sci. 2003;58:P112-28.

[4] Oliva-Moreno J, Trapero-Bertran M, Peña-Longobardo LM, Del Pozo-Rubio R. "The Valuation of Informal Care in Cost-of-Illness Studies: A Systematic Review". Pharmacoeconomics. 2017; 35: 331-345..

[5] Pickard L, King D. Informal care supply and demand in Europe. In: Geerts. J, Willeme P, Mot E, editors. Long-term care use and supply in Europe: projections for Germany, the Netherlands, Spain and Poland.Brussels: European Network of Economic Policy Research Institutes (ENEPRI). 2012.Research Report 116:114-23.

[6] Lyons JG, Cauley JA, Fredman L. The Effect of transitions in caregiving status and intensivity on perceived stress among 992 female caregivers and noncaregivers. J Gerontol A Biol Sci Med Sci. 2015 Aug;70(8):1018-23.

[7] Susan K.Roepke, Matthew Allison, Roland Von Ka Nel, Brent T. Mausbach, et al. "Relationship between chronic stress and carotid intima-media thickness (IMT) in elderly Alzheimer's disease caregivers". Stress, March 2012; 15(2): 121-129.

[8] Association of psychosocial risk factors with risk of acute myocardial infarction in 11119 cases and 13648 controls from 52 countries. (the INTERHEART study): case-control study. The Lancet; Sep 11-Sep 17, 2004; 364, 9438; Health \& Medical Collection. 
Sira Aguiló, Beatriz Lopez-Barbeito, Esther García, Jordi Aguiló. Stress Management in Primary Caregivers: A

Health Challenge. Transactions on Machine Learning and Artificial Intelligence, Vol 5 No 4 August (2017);

p: $558-564$

[9] Haug, M. R., Ford, A. B., Stange, K. C., Noelker, L. S., \& Gaines, A. D. (1999). Effect of giving care on caregivers' health. Research on Aging, 21(4), 515-538.

[10] Janice K. Kiecolt-Glaser, Lymama Mc Guire, Theodore F Robles, Ronald Glaser. Psychoneuroimmunology: Psychological Influences on Immune Function and Health. Journal of Consulting and Clinical Psychology 2002; vol 70; 537-547.

[11] Ashi, S., Girona, G. y Ortuño, M. A. (1995). Sindrome del cuidador. Rehabilitación, 29, 465-468.

[12] M.J. Gómez-Ramos, F.M. Gonzáñez-Valverde. Caregivers of dementia patients: application of the Caregiver Burden Scale. Rev Esp Geriatr Gerontol 2004;39:154-9.

[13] Schulz R, Beach SR. Caregiving as a risk factor for mortality: the Caregiver Health Effects Study. JAMA. 1999;282:2215-9.

[14] Zarit SH, Reever KE, Bach-Peterson J. Relatives of the impaired elderly: correlates of feelings of burden. Gerontologist. 1980;20:649-55.

[15] http://blog.hospitalclinic.org/es/2014/06/presentacion-del-programa-infosa-de-apoyo-a-cuidadores-depersonas-dependientes/.

[16] Cheryl L. Kern-Buell, Angele Mc Grady, Philip B. Conran, Lois A. Nelson. Asthma severity, Psichophysiological indicators of arousal, and inmmune function in asthma patients undergoing biofeedback-assisted relaxation. Applied Psychophysiology and biofeedback. June 2000, vol 25. Issue 2, 79-91.

[17] Juan Manuel Vázquez Goñi et al, "Reducing the stress of food processing workers through respiratory biofeedback". Revista Cubana de salud y trabajo 2011; 12 (1): 39-44.

[18] Hernando A, Lazaro J, Gil E, Arza A, Garzón-Rey JM et al. Inclusion of respiratory frequency information in Heart Rate Variability for a better analysis of Stress.IEEE JBHI2016; 20 (4): 1016-1025

[19] Aguilo J, Ferrer-Salvans P, Garcia-Rozo A, et al. Project ES3: attempting to quantify and measure the level of stress. Rev Neurol. 2015;61(9):405-415.

[20] Dunn SA Bonner PN, Lewis SL, Meize-Grochowski R. Quality of life for spouses of CAPD patients. ANNA Journal. 1994;21:237-247. 